\title{
Germline mutations in the alternative pathway of complement predispose to HELLP syndrome
}

\author{
Arthur J. Vaught, ${ }^{1}$ Evan M. Braunstein, ${ }^{2}$ Jagar Jasem,${ }^{2}$ Xuan Yuan, ${ }^{2}$ Igor Makhlin, ${ }^{2}$ \\ Solange Eloundou, ${ }^{1}$ Andrea C. Baines, ${ }^{2}$ Samuel A. Merrill, ${ }^{2}$ Shruti Chaturvedi, ${ }^{2}$ Karin Blakemore, ${ }^{1}$ \\ C. John Sperati, ${ }^{3}$ and Robert A. Brodsky² \\ 'Division of Maternal Fetal Medicine, Department of Gynecology and Obstetrics, 2Division of Hematology, Department \\ of Medicine, and ${ }^{3}$ Division of Nephrology, Department of Medicine, Johns Hopkins University School of Medicine, \\ Baltimore, Maryland, USA.
}

BACKGROUND. HELLP (hemolysis, elevated liver enzymes, and low platelets) syndrome is a severe variant of hypertensive disorders of pregnancy affecting approximately $1 \%$ of all pregnancies, and has significant maternal and fetal morbidity. Previously, we showed that upregulation of the alternative pathway of complement (APC) plays a role in HELLP syndrome. We hypothesize that HELLP syndrome follows a 2-hit disease model similar to atypical hemolytic uremic syndrome (aHUS), requiring both genetic susceptibility and an environmental risk factor. Our objective was to perform a comparative analysis of the frequency of APC activation and germline mutations in affected women and to create a predictive model for identifying HELLP syndrome.

METHODS. Pregnant women with HELLP syndrome, and healthy controls after 23 weeks of gestation were recruited, along with aHUS and thrombotic thrombocytopenic purpura participants. We performed a functional assay, the $\mathrm{mHam}$, and targeted genetic sequencing in all groups.

RESULTS. Significantly more participants with rare germline mutations in APC genes were present in the HELLP cohort compared with controls ( $46 \%$ versus $8 \%, P=0.01$ ). In addition, significantly more HELLP participants were positive for the mHam when compared with controls (62\% versus $16 \%, P=0.009$ ). Testing positive for both a germline mutation and the $\mathrm{mHam}$ was highly predictive for the diagnosis of HELLP syndrome.

CONCLUSION. HELLP syndrome is characterized by both activation of the APC and frequent germline mutations in APC genes. Similar to aHUS, treatment via complement inhibition to mitigate maternal and fetal morbidity and mortality may be possible.

FUNDING. National Heart Lung and Blood Institute grants T32HL007525 and R01HL133113.

Authorship note: AJV and EMB contributed equally to this work.

Conflict of interest: RAB is currently serving as an Alexion Pharmaceuticals Scientific Advisory Board member, an Achillion Pharmaceuticals Scientific Advisor (or consultant), and an Apellis Pharmaceuticals Scientific Advisor (or consultant).

Submitted: December 8, 2017

Accepted: February 7, 2018

Published: March 22, 2018

Reference information:

JCI Insight. 2018;3(6):e99128. https:// doi.org/10.1172/jci.insight.99128

\section{Introduction}

Preeclampsia is a devastating multisystem disorder of pregnancy that occurs in $3 \%-5 \%$ of all pregnancies, manifesting as hypertension with or without proteinuria and/or end-organ damage. Notably, not only does preeclampsia account for maternal morbidity, but it also accounts for $30 \%$ of all preterm deliveries, which results in neonatal intensive care unit admissions, increased health care costs, severe neonatal morbidity, and neonatal mortality (1-3). HELLP (hemolysis, elevated liver enzymes, and low platelets) syndrome is the most severe variant of this disorder, and affects approximately $1 \%$ of all pregnancies (4). While thought to be due to endothelial cell dysfunction, the precise etiology of both preeclampsia and HELLP syndrome remains unclear, resulting in treatment with supportive regimens such as fetal monitoring, steroids for fetal lung maturity, magnesium for seizure prophylaxis, management of hypertension and ultimately delivery that results in iatrogenic preterm birth $(1,5-7)$. Furthermore, the diagnostic criteria for HELLP syndrome are ambiguous, with 2 distinct sets of criteria (Mississippi and Tennessee) based on clinical testing that can be confused by differences in laboratory assays (8). 
Complement plays a crucial role in host immunity secondary to the opsonization of pathogens, the recruitment and activation of inflammatory cells, and the initiation of membrane attack complexes (MACs) $(9,10)$. It consists of an enzymatic cascade of over 30 proteins that are activated by the classical pathway, the lectin pathway, and the alternative pathway (9). While the classical pathway depends on antigen-antibody complexes (e.g., lupus) for activation, the alternative pathway of complement (APC) is antibody independent and has various triggers including infection, trauma, and pregnancy (11). In disease states, dysregulation of the APC is frequently secondary to inactivation of regulatory proteins, resulting in amplification loops that increase proinflammatory cytokines and activation of C3 and C5, leading to an increase in formation of MACs and subsequent endothelial damage.

Although integral in host survival, the overactivation of complement can cause highly morbid diseases, exemplified by atypical hemolytic uremic syndrome (aHUS). aHUS is caused by APC dysregulation and presents with signs of thrombocytopenia, hemolytic anemia, acute kidney injury, hypertension, and neurologic abnormalities, a clinical presentation phenotypically similar to HELLP syndrome $(12,13)$. Heterozygous germline mutations in genes that function in the APC are found in approximately 50\% of aHUS patients, leading to loss of function of complement inhibitors or occasionally gain of function in complement activators. Mutations in APC genes have also been identified in small cohorts of patients with preeclampsia and HELLP syndrome (14-21). While genetic alterations confer susceptibility to disease in aHUS, an additional risk factor, or trigger, is required for disease onset (12). Importantly, treatment with terminal complement inhibitors such as eculizumab are effective in treating aHUS (22).

Previously, we assessed activation of the APC in patients with HELLP syndrome using the modified Ham (mHam) test (13). This assay measures the ability of a patient's serum to induce death of cells sensitive to complement activity due to a lack of glycosylphosphatidylinositol-modified (GPI-modified) proteins. Without GPI, the cells cannot anchor crucial complement regulator proteins of the APC to their membrane, such as CD55 and CD59, and are unable to regulate complement activation (20). Thus, the mHam is a functional assay that can be used to delineate diseases of APC dysregulation, and has been validated in its ability to distinguish aHUS from thrombotic microangiopathies (TMAs) that have similar clinical presentations but differ in pathophysiology $(13,20)$. We previously reported that like aHUS, a majority of HELLP syndrome patients display dysregulation of the APC in their serum, evidenced by increased cell killing in the mHam assay (13).

Based on our data, we hypothesized that HELLP syndrome follows a 2-hit disease model similar to aHUS, requiring both a germline susceptibility mutation as well as an environmental risk factor (pregnancy). Here, we show that the APC is more likely to be active in the serum of patients with HELLP syndrome compared with healthy pregnant females. In addition, we find that patients with HELLP syndrome harbor germline mutations in APC genes at a similar prevalence to that seen in aHUS. We envision this information to be useful in identifying women that have APC dysregulation that may respond to complement inhibition, much like other diseases of the APC.

\section{Results}

Participant demographics. To test our hypothesis, women with HELLP syndrome, partial HELLP syndrome, and healthy pregnant controls were recruited from 2015 to 2016. Our cohort consisted of 13 participants with HELLP syndrome (H1-H13, Table 1), 14 participants with partial HELLP syndrome (PH1-PH14, Table 2), and 19 healthy pregnant controls (HPC1-HPC19, Table 3). In order to compare these cohorts to patients with TMA, we also recruited 18 participants diagnosed with aHUS (aHUS1-aHUS18, Table 4), and 8 participants diagnosed with thrombotic thrombocytopenic purpura (TTP1-TTP8, Table 4). The participant demographics among HELLP, partial HELLP, and HPC cohorts did not differ in body mass index (BMI), race, or parity. However, the HELLP group had a statistically significant younger age when compared with the HPC group (27 versus 32 years, $P<0.030$ ), but not when compared with partial HELLP syndrome. We also found that the mean estimated gestational age (EGA) of HELLP participants was significantly younger compared with the HPC group (30 versus 37 weeks, $P<0.001$ ).

HELLP participants had a significantly greater incidence of adverse maternal and/or fetal events compared with both partial HELLP and HPC groups. Of the HELLP group participants, 7 of 13 (54\%) had adverse maternal events. One woman was admitted to the intensive care unit and required renal replacement therapy for acute renal failure. Seven women needed blood product transfusions, and one participant had an eclamptic seizure. In addition, 7 of 13 women (54\%) had adverse fetal events in the HELLP 
Table 1. HELLP syndrome participants

\begin{tabular}{|c|c|c|c|c|c|c|c|c|c|c|c|c|c|c|c|c|}
\hline ID & $\begin{array}{l}\text { EGA } \\
\text { (wk) }\end{array}$ & Race & $\begin{array}{l}\text { Age } \\
\text { (yr) }\end{array}$ & BMI & $P$ & SBP/DBP & $\begin{array}{c}\mathrm{Cr} \\
(\mathrm{mg} / \mathrm{dl})\end{array}$ & $\begin{array}{l}\text { Highest AST } \\
\text { (IU/I) }\end{array}$ & $\begin{array}{l}\text { T Highest ALT } \\
\text { (IU/I) }\end{array}$ & $\begin{array}{l}\text { Lowest } \\
\text { Platelets } \\
\left(\mathrm{mm}^{3}\right)\end{array}$ & $\mathrm{Pr} / \mathrm{Cr}$ & $\begin{array}{c}\text { Highest } \\
\text { LDH } \\
\text { (IU/I) }\end{array}$ & $\begin{array}{c}\text { mHAM } \\
(\%)\end{array}$ & $\begin{array}{l}\text { Germline } \\
\text { Variant }\end{array}$ & $\begin{array}{c}\text { Adverse } \\
\text { Maternal } \\
\text { Events }\end{array}$ & $\begin{array}{c}\text { Adverse } \\
\text { Fetal Events }\end{array}$ \\
\hline $\mathrm{H} 1$ & 31.2 & Black & 28 & 34.5 & 0 & $169 / 103$ & 0.7 & 64 & 63 & 85,000 & 0.43 & 431 & 32 & $\begin{array}{c}\text { CFHR1 } \\
\text { p.L322Rfs*50 }\end{array}$ & None & FGR \\
\hline $\mathrm{H} 2$ & 26.4 & Black & 21 & 26.9 & 0 & $140 / 80$ & 0.9 & 321 & 287 & 50,000 & 3.20 & 867 & 32 & C3 p.C542D & None & FGR \\
\hline H3 & 34.2 & Black & 33 & 28.3 & $>2$ & $204 / 121$ & 6.5 & 520 & 86 & 21,000 & 4.50 & 2,030 & 29 & $\begin{array}{c}\text { del(CFHR1) } \\
\text { homozygous }\end{array}$ & $\begin{array}{l}\text { MV, RRT, } \\
\text { PLT, RBC }\end{array}$ & None \\
\hline $\mathrm{H} 4$ & 33 & White & 35 & 22.8 & 0 & $141 / 80$ & 0.7 & 1,163 & 749 & 28,000 & 1.46 & 1,461 & 21 & $\begin{array}{c}\text { CFHR5 } \\
\text { p.K562Nfs*24 }\end{array}$ & None & None \\
\hline H5 & 24 & White & 26 & 30 & $>1^{A}$ & $150 / 72$ & 0.7 & 167 & 145 & 100,000 & 0.24 & 574 & 8 & $\begin{array}{l}\text { del(CFHR3- } \\
\text { CFHR1) } \\
\text { homozygous }\end{array}$ & None & Termination \\
\hline $\mathrm{H} 6$ & 17.2 & Black & 24 & 23.8 & 0 & $165 / 109$ & 1.2 & 84 & 65 & 77,000 & 0.32 & 540 & 33 & None & PLT & Termination \\
\hline $\mathrm{H} 7$ & 38 & White & 32 & 47.1 & 0 & $180 / 100$ & 0.9 & 420 & 308 & 43,000 & 0.39 & 591 & 30 & None & RBC, PLT & None \\
\hline $\mathrm{H} 8$ & 24.2 & Black & 22 & 33.6 & 0 & $180 / 100$ & 1.3 & 338 & 170 & 52,000 & 5.04 & 2,015 & 23 & None & $\begin{array}{l}\text { RBC, PLT, } \\
\text { Eclampsia }\end{array}$ & FGR \\
\hline H9 & 36 & White & 23 & 29.3 & 0 & $166 / 98$ & 0.6 & 243 & 181 & 42,000 & 12.35 & 581 & 23 & None & PLT & FGR \\
\hline $\mathrm{H} 10$ & 32.1 & Black & 15 & 16.3 & 0 & $230 / 110$ & 0.6 & 114 & 81 & 42,000 & $\mathrm{~N} / \mathrm{A}$ & 612 & 11 & NS & RBC & None \\
\hline $\mathrm{H} 11$ & 34.2 & Black & 19 & 24 & 0 & $191 / 84$ & 0.6 & 201 & 101 & 63,000 & 6.93 & 325 & 10 & None & None & None \\
\hline $\mathrm{H} 12$ & 22.2 & Black & 45 & 33.7 & $>2$ & $155 / 90$ & 0.7 & 131 & 125 & 68,000 & 9.77 & 503 & 0 & None & None & $\begin{array}{c}\text { FGR } \\
\text { Termination }\end{array}$ \\
\hline $\mathrm{H} 13$ & 37.2 & White & 27 & 36.2 & 0 & $180 / 91$ & 0.6 & 695 & 423 & 19,000 & 0.19 & 805 & 0 & NS & PLT & None \\
\hline
\end{tabular}

${ }^{A}$ History of prior HELLP (hemolysis, elevated liver enzymes, and low platelets) syndrome or eclampsia in previous pregnancy. EGA, estimated gestational age; wk, weeks; yr, years; BMI, body mass index; BP, blood pressure; P, parity; SBP, systolic blood pressure (mmHg); DBP, diastolic blood pressure (mmHg); $\mathrm{Cr}$, creatinine; AST, aspartate aminotransferase; ALT, alanine aminotransferase; $\mathrm{Pr} / \mathrm{Cr}$, protein/creatinine ratio; LDH, lactate dehydrogenase; mHam, modified Ham (positive for modified Ham > 20.5\%); FGR, fetal growth restriction; MV, mechanical ventilation; RRT, renal replacement therapy; NS, not sequenced; RBC, red blood cell transfusion; PLT, platelet transfusion.

syndrome group. Three participants terminated their pregnancies secondary to the diagnosis of HELLP syndrome in the setting of a nonviable fetus (less than 23 weeks and/or estimated fetal weight less than 500 grams). The other 5 fetuses survived but were diagnosed with fetal growth restriction (FGR), which manifested in the third trimester.

There was one adverse maternal outcome in the partial HELLP group, which was an eclamptic seizure. Further, 4 of 14 participants (29\%) experienced the adverse fetal event of FGR. There were no fetal terminations in the partial HELLP group. In our HPC cohort, there was one participant who was later diagnosed with preeclampsia (not HELLP or partial HELLP syndrome). At the time of recruitment, the participant was normotensive without signs or symptoms of preeclampsia and had an EGA of 38 weeks. She was diagnosed with preeclampsia at 39 weeks and 5 days and this necessitated induction of labor. There was one adverse fetal outcome, which was FGR in the HPC group. All HELLP or partial HELLP syndrome resolved postpartum or after termination in all cases, and no cases of pregnancy-associated aHUS or TTP were observed.

Complement activation in HELLP and aHUS. The mHam assay was performed for all participants with available serum samples in order to assess activation of the APC. A positive test is defined as greater than $20.5 \%$ cell killing, based on our previous study suggesting that HELLP patients are positive in the mHam assay (13). In this analysis, 8 of 13 HELLP participants (62\%) were positive, compared with only 2 of 18 participants (11\%) in the HPC group (Figure 1). In addition, only 3 of $14(21 \%)$ partial HELLP participants were positive in the mHam assay. For participants with aHUS, 15 of $17(88 \%)$ were positive, while only 1 of 7 participants (14\%) with TTP were positive, confirming as expected that the mHam is a useful discriminator between these 2 phenotypes (20).

While the mHam assesses complement activation as a measure of relative cell death, the magnitude of a result above the $20.5 \%$ threshold has unclear significance and has not been found to be associated with clinical outcomes. Thus, statistical analysis was performed using the mHam as a binary measure (positive or negative). When compared with the HPC group, HELLP syndrome participants were significantly more likely to test positive for the mHam assay (62\% versus $11 \%, P=0.006)$ (Table 5 ). We also 
Table 2. Partial HELLP syndrome participants

\begin{tabular}{|c|c|c|c|c|c|c|c|c|c|c|c|c|c|c|c|c|}
\hline ID & $\begin{array}{l}\text { EGA } \\
\text { (wk) }\end{array}$ & Race & $\begin{array}{l}\text { Age } \\
\text { (yr) }\end{array}$ & BMI & $P$ & SBP/DBP & $\begin{array}{c}\mathrm{Cr} \\
(\mathrm{mg} / \mathrm{dl})\end{array}$ & $\begin{array}{c}\text { Highest AST } \\
\text { (IU/I) }\end{array}$ & $\begin{array}{c}\text { Highest ALT } \\
\text { (IU/I) }\end{array}$ & $\begin{array}{c}\text { Lowest } \\
\text { Platelets } \\
\left(\mathrm{mm}^{3}\right)\end{array}$ & $\mathrm{Pr} / \mathrm{Cr}$ & $\begin{array}{c}\text { Highest LDH } \\
\text { (IU/I) }\end{array}$ & $\begin{array}{c}\text { mHAM } \\
(\%)\end{array}$ & $\begin{array}{l}\text { Germline } \\
\text { Variant }\end{array}$ & $\begin{array}{l}\text { Adverse } \\
\text { Maternal } \\
\text { Events }\end{array}$ & $\begin{array}{c}\text { Adverse } \\
\text { Fetal } \\
\text { Events }\end{array}$ \\
\hline $\mathrm{PH} 1$ & 32.4 & Black & 24 & 25.2 & $>1^{A}$ & $169 / 98$ & 0.6 & 99 & 14 & 176,000 & 0.67 & N/A & 7 & $\begin{array}{l}\text { C3 p.P890H, } \\
\text { CFI p.1416L, } \\
\text { del(CFHR3- } \\
\text { CFHR1) } \\
\text { homozygous }\end{array}$ & None & FGR \\
\hline $\mathrm{PH} 2$ & 30.6 & Black & 25 & 32.7 & $>2$ & $180 / 123$ & 2.0 & 51 & 45 & 106,000 & 6.43 & 492 & 6 & $\begin{array}{c}\text { C3 p.D1457H, } \\
\text { del(CFHR3- } \\
\text { CFHR1) } \\
\text { homozygous }\end{array}$ & None & None \\
\hline $\mathrm{PH} 3$ & 34 & Black & 29 & 35.5 & 0 & $176 / 91$ & 1.0 & 72 & 72 & 164,000 & 12.2 & 430 & 4 & $\begin{array}{c}\text { del(CFHR3- } \\
\text { CFHR1) } \\
\text { homozygous }\end{array}$ & None & FGR \\
\hline $\mathrm{PH} 4$ & 27 & White & 36 & 41.7 & 0 & $200 / 80$ & 0.7 & 78 & 78 & 293,000 & 1.52 & 529 & 37 & None & None & None \\
\hline PH5 & 30.4 & Asian & 26 & 27.1 & 0 & $190 / 110$ & 0.5 & 55 & 66 & 176,000 & 0.59 & 184 & 27 & None & None & FGR \\
\hline PH6 & 28.1 & Black & 25 & 32.4 & $>1$ & $173 / 103$ & 0.7 & 93 & 90 & 130,000 & 3.00 & 554 & 22 & None & Eclampsia & FGR \\
\hline $\mathrm{PH} 7$ & 37.5 & White & 41 & 34 & 0 & $157 / 100$ & 0.8 & 65 & 89 & 140,000 & 0.13 & 161 & 20 & None & None & None \\
\hline $\mathrm{PH} 8$ & 37.5 & Black & 30 & 42.3 & $>2$ & $171 / 90$ & 1.1 & 792 & 841 & 294,000 & 0.45 & 1,124 & 18 & None & None & None \\
\hline PH9 & 39.2 & Black & 34 & 52.4 & 0 & $183 / 84$ & 0.9 & 134 & 74 & 237,000 & 0.63 & 284 & 12 & None & None & None \\
\hline PH10 & 37.2 & Black & 29 & 39.1 & 0 & $194 / 106$ & 0.8 & 28 & 23 & 222,000 & 0.35 & 434 & 11 & None & None & None \\
\hline PH11 & 31.2 & White & 31 & 19.6 & 0 & $165 / 71$ & 0.6 & 22 & 22 & 84,000 & 0.86 & 228 & 6 & None & None & None \\
\hline $\mathrm{PH} 12$ & 32.6 & White & 33 & 28.4 & $>1$ & $145 / 77$ & 1.1 & 181 & 189 & 316,000 & 6.23 & 222 & 3 & None & None & None \\
\hline PH13 & 39.3 & Black & 30 & 35.5 & $>1$ & $180 / 103$ & 1 & 76 & 88 & 249,000 & 0.20 & 480 & 3 & None & None & None \\
\hline $\mathrm{PH} 14$ & 34.4 & Asian & 27 & 27.2 & 0 & $158 / 97$ & 0.6 & 69 & 91 & 261,000 & 8.71 & 317 & 0 & None & None & None \\
\hline
\end{tabular}

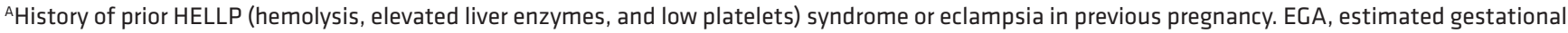
age; wk, weeks; yr, years; BMI, body mass index; BP, blood pressure; P, parity; SBP, systolic blood pressure (mmHg); DBP, diastolic blood pressure (mmHg); $\mathrm{Cr}$, creatinine; AST, aspartate aminotransferase; ALT, alanine aminotransferase; $\mathrm{Pr} / \mathrm{Cr}$, protein/creatinine ratio; LDH, lactate dehydrogenase; mHam, modified Ham (positive for modified Ham > 20.5\%); FGR, fetal growth restriction.

generated a control cohort for a TMA during pregnancy by combining the HPC and TTP groups, as both are expected to have normal complement regulation, and the TTP participants are a confirmed negative control in our previous study (20). HELLP participants were significantly more likely to test positive in the mHam assay ( $62 \%$ versus $16 \%, P=0.009)$ compared with the composite control cohort. This was also true for aHUS patients compared with this cohort $(88 \%$ versus $16 \%, P<0.001)$. Conversely, mHam positivity in the HELLP syndrome and aHUS groups was not significantly different. These data indicate that HELLP syndrome, similar to aHUS, is marked by activation of complement.

Identification of rare germline variants in HELLP syndrome. Approximately $50 \%$ of aHUS patients harbor germline mutations in genes that regulate the APC; thus, we performed targeted sequencing of participant DNA to identify germline variants in 9 genes known to have a functional role in the APC. To minimize the contribution of likely benign germline polymorphisms, only rare heterozygous germline variants with a minor allele frequency less than 0.01 (1\%) were included in our analysis (detailed in methods). In addition, homozygous deletion of CFHR 1 and CFHR3, reported to occur in approximately $2 \%$ of the population, was included in our analysis owing to its association with aHUS and likely functional significance (21). In our pregnant cohorts, 2 participants in the HELLP syndrome group and 2 participants in the HPC group did not give consent for gene mutation testing. We identified a rare germline variant or homozygous deletion in 5 of 11 HELLP participants (46\%), compared with only 3 of 14 partial HELLP participants (21\%), and 2 of 17 participants (12\%) in the HPC group (Tables 1-3 and Figure 2). Further, in our TMA cohorts, germline variants were found in 10 of 18 participants with aHUS (56\%), while no variants were identified any of the 8 TTP participants (Table 4). When compared with the HPC group alone, there was a nonsignificant trend towards the presence of a genetic variant in the HELLP syndrome cohort ( $46 \%$ versus $12 \%, P=0.07)$. When compared with the composite controls (combined HPC and TTP groups), HELLP patients were significantly more likely to have a rare germline variant in an APC gene ( $46 \%$ versus $8 \%, P=0.01)$. In addition, there was no difference between the HELLP syndrome and aHUS cohorts for the presence of a genetic variant (Table 6). 
Table 3. Healthy pregnant control (HPC) participants

\begin{tabular}{|c|c|c|c|c|c|c|c|c|c|c|}
\hline ID & EGA & Race & Age & BMI & $P$ & SBP/DBP & mHam (\%) & $\begin{array}{l}\text { Germline } \\
\text { Variant }\end{array}$ & $\begin{array}{l}\text { Adverse } \\
\text { Maternal } \\
\text { Events }\end{array}$ & $\begin{array}{c}\text { Adverse Fetal } \\
\text { Events }\end{array}$ \\
\hline $\mathrm{HPC1}{ }^{\mathrm{A}}$ & 37.3 & Black & 34 & 36.6 & $>1$ & $128 / 71$ & 8 & $\begin{array}{l}\text { CFH p.H371N, } \\
\text { CFHR5 p.S78P, } \\
\text { CFHR5 p.Y279N }\end{array}$ & None & None \\
\hline HPC2 & 38 & Black & 27 & 29.8 & 0 & $128 / 85$ & 4 & CFHR3 p.C15Y & None & None \\
\hline HPC3 & 38.1 & White & 31 & 26.7 & $>1$ & $122 / 76$ & 100 & None & None & None \\
\hline HPC5 & 39.1 & Other & 32 & 37.6 & $>3$ & $124 / 83$ & 16 & None & None & None \\
\hline HPC6 & 39 & Asian & 36 & 25.4 & $>2$ & $105 / 64$ & 15 & None & None & None \\
\hline HPC7 & 38 & White & 35 & 35.1 & 0 & $170 / 100$ & 14 & None & Preeclampsia & None \\
\hline HPC8 & 38 & Black & 38 & 28.6 & $>1$ & $121 / 87$ & 13 & None & None & None \\
\hline HPC9 & 37.1 & White & 35 & 22.8 & $>1$ & $119 / 67$ & 12 & None & None & None \\
\hline HPC10 & 36 & Black & 32 & 32 & $>3$ & $116 / 61$ & 9 & None & None & None \\
\hline HPC14 & 37 & Black & 31 & 40.8 & $>3$ & $133 / 62$ & 5 & None & None & None \\
\hline HPC15 & 39.3 & White & 37 & 28.1 & 0 & $121 / 84$ & 5 & NS & None & None \\
\hline HPC16 & 36.2 & White & 30 & 27.6 & $>2$ & $127 / 76$ & 3 & None & None & None \\
\hline HPC17 & 38.6 & White & 33 & 28.6 & 0 & $118 / 75$ & 3 & NS & None & None \\
\hline HPC18 & 37 & White & 36 & 20.5 & 0 & $135 / 85$ & 1 & None & None & None \\
\hline HPC19 & 38 & White & 28 & 37.7 & $>1$ & $110 / 73$ & $\mathrm{~N} / \mathrm{A}$ & None & None & FGR \\
\hline
\end{tabular}

${ }^{A}$ History of sickle cell trait. EGA, estimated gestational age; wk, weeks; yr, years; BMI, body mass index; BP, blood pressure; P, parity; SBP, systolic blood pressure ( $\mathrm{mmHg}$ ); DBP, diastolic blood pressure (mmHg); mHam, modified Ham (positive for modified Ham > 20.5\%); FGR, fetal growth restriction; NS, not sequenced.

Detailed characteristics of all identified genetic variants, along with pathogenicity prediction outcomes for single-nucleotide variants (SNVs) via 3 different algorithms, are provided in Table 7. There were 2 HELLP participants with frameshift mutations, assumed to be loss of function, and 1 participant with a missense mutation predicted to be pathogenic in 2 out of 3 algorithms. Each of these participants also had a positive mHam test. Another HELLP participant with a positive mHam test harbored a homozygous deletion of the CFHR1 gene. There was one HELLP participant with a negative mHam assay and a germline variant. This individual (H5) was found to have homozygous deletion of both CFHR1 and CFHR3 and carried a history of HELLP syndrome in a prior pregnancy. Interestingly, homozygous deletion of $C F H R 1$ and CFHR3 was found in 3 participants with partial HELLP, all with negative mHam tests. One of these participants (PH1) also had a history of HELLP syndrome in a prior pregnancy. Of note, PH1 was found to have 2 additional missense variants in other APC genes as well. There were 2 HPC participants identified with rare genetic variants, both negative in the mHam assay. One individual (HPC1) carried a diagnosis of sickle cell trait and $\alpha$-thalassemia and harbored multiple missense mutations, with one predicted to be pathogenic by all 3 algorithms. By comparison, all aHUS participants with identified germline variants were also found to be positive in the mHam assay. Two participants were found to have deletions of both CFHR1 and CFHR3, while 1 participant harbored a deletion of $C F H R 1$ only. Deletion of $C F H R$ genes were not identified in any HPC or TTP participants, supporting the functional significance of this variant. However, this variant by itself does not appear to sufficiently activate the APC in order to produce a positive mHam test.

\section{Discussion}

In this study, we demonstrate that HELLP syndrome is part of the phenome that results from germline mutations in genes that regulate the APC. Using phenotypic, functional (mHam assay demonstrating impaired complement regulation), and genetic (mutations in genes that regulate the APC) data we found striking similarities to aHUS. Our genetic analysis was limited to rare germline variants in order to increase the likelihood of identifying pathogenic mutations that alter the function of APC genes. Using this strict criterion, significantly more APC germline mutations were identified in the HELLP syndrome cohort compared with 


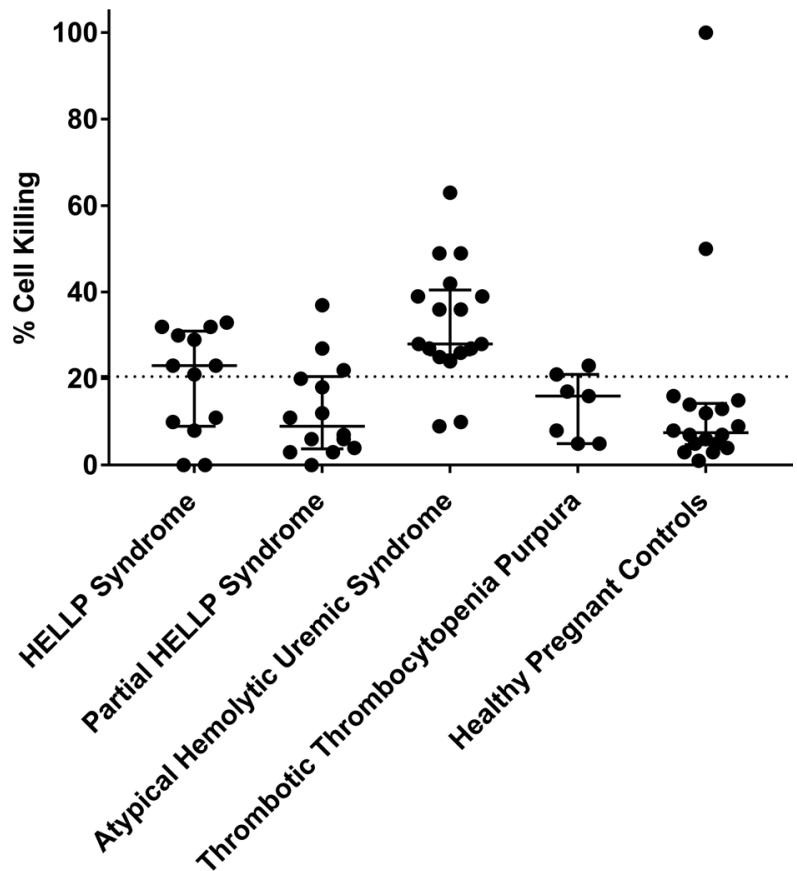

Figure 1. Modified Ham (mHam) results among all participant groups. Each participant is represented as a black circle, and cell killing in the mHAM assay is displayed as a continuous variable for each participant group. The median and interquartile range of $25 \%-75 \%$ is depicted for each group. Statistical analysis demonstrating significant differences between groups using the mHam as a binary measure (positive vs. negative) is shown in Table 6. A positive mHam is defined as cell killing of greater than $20.5 \%$ (dashed black horizontal line). HELLP, hemolysis, elevated liver enzymes, and low platelets.

\section{Participant Groups}

controls ( $46 \%$ versus $8 \%, P=0.01$ ), while no difference was found when compared with aHUS. We also confirmed our previous data demonstrating that significantly more HELLP participants test positive in the mHam assay compared with controls $(62 \%$ versus $16 \%, P=0.009)$. Further, we did not identify any individuals in our control groups that tested positive for both the mHam assay and a germline variant ( 0 of 23 HPC and TTP participants, compared with 4 of 11 HELLP participants). Conversely, both tests were negative in 17 of 23 control participants and only 2 of 11 HELLP participants. Thus, this combination of testing may identify individuals with HELLP syndrome who could potentially benefit from therapeutic intervention.

Our findings agree with previous studies suggesting that complement plays an important role in the pathophysiology of HELLP. The APC activation fragment $\mathrm{Bb}$ was found to be a predictor of acquiring preeclampsia (23). In addition, a prospective study of 40 pregnant women with lupus and/or antiphospholipid antibodies who developed preeclampsia identified 7 (17.5\%) that had germline heterozygous mutations likely to be deleterious in $M C P, C F I$, or $C F H$ (14). Other studies have performed targeted sequencing of various APC genes in patients with HELLP syndrome and identified heterozygous mutations in $9 \%$ to $36 \%$ of cases $(18,19)$.

The major limitation of this study is the small sample sizes of the participant groups. Further, multiple HELLP and partial HELLP participants tested negative for APC activation in the mHam assay. The most likely explanation for this finding is that the pathophysiology of HELLP syndrome (and partial HELLP even more so) is due to multiple factors. However, this study builds on our previous data and that of others demonstrating that a significant subset of HELLP syndrome is due to a reduced ability to regulate the APC, analogous to aHUS $(13,18,20)$. Indeed, the clinical manifestations of hypertension, renal insufficiency, thrombocytopenia, elevated lactate dehydrogenase, elevated aspartate aminotransferase, and even the presence of schistocytes are common to both disorders. Roughly $50 \%$ of aHUS patients harbor germline mutations, usually heterozygous, that increase susceptibility of endothelial cells to injury from activated complement $(12,15,16)$. These patients are often asymptomatic for decades until they encounter a strong complement trigger such as major surgery, infection, malignancy, autoimmunity, or pregnancy. In HELLP syndrome, pregnancy is the likely trigger (11). Previous studies have shown that complement proteins such as $\mathrm{CFH}$ and $\mathrm{C} 5 \mathrm{~b}-9$, as well as associated contributors of increased complement (e.g., C-reactive protein) are significantly increased during pregnancy, possibly explaining why phenotypic manifestations of HELLP syndrome almost exclusively occur in the late second or third trimester $(9,24-27)$. This may also explain why 2 cases in the HPC cohort tested positive in the mHam assay. 


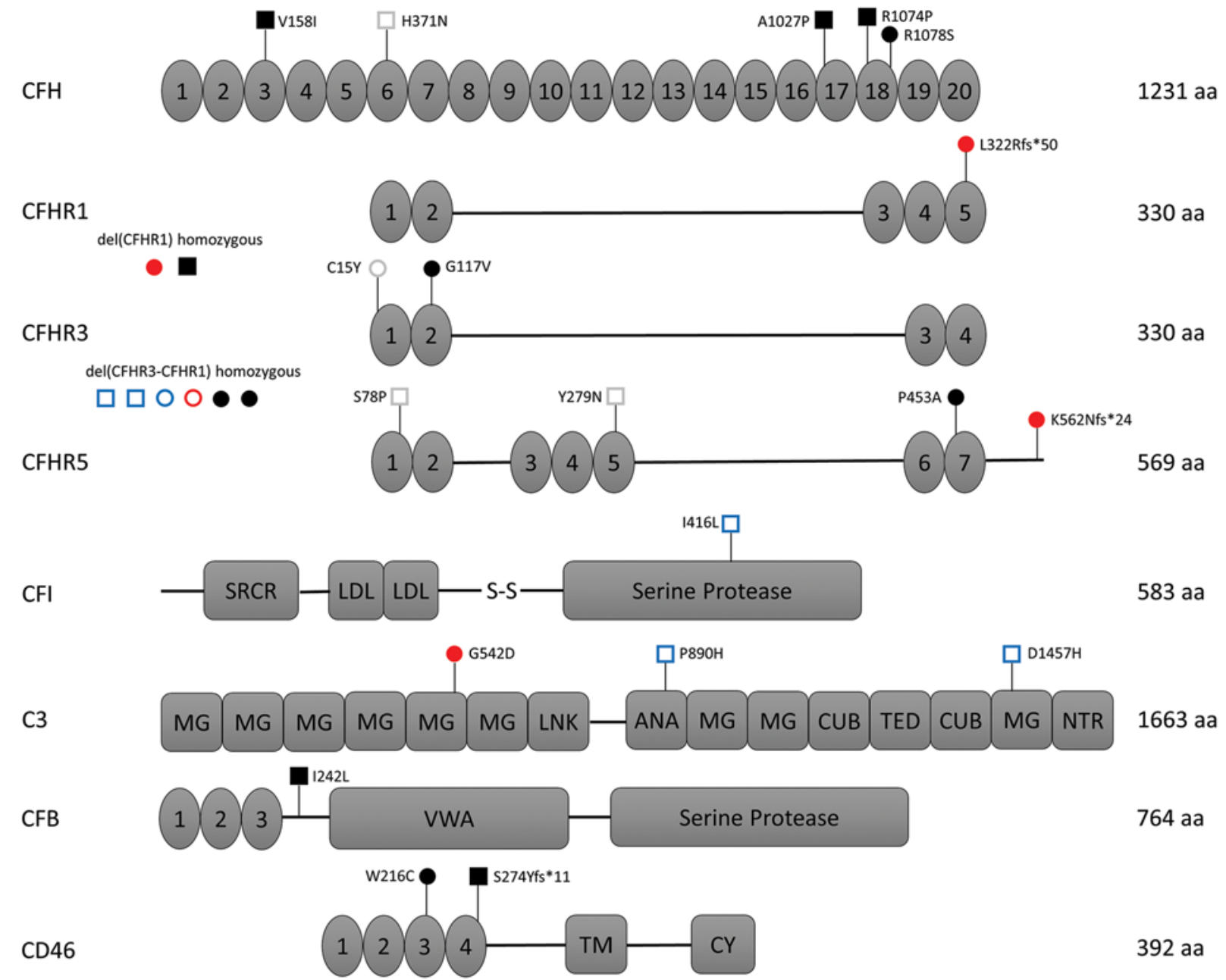

\begin{tabular}{|ll|}
\hline \multicolumn{2}{|l|}{ Legend } \\
aHUS \\
HELLP \\
partial HELLP \\
pregnant control \\
\hline
\end{tabular}

Figure 2. Correlation of germline variants in complement proteins with functional testing. Each genomic variant is identified by its amino acid consequence along with the result of modified $\mathrm{Ham}(\mathrm{mHam})$ testing in that patient. A filled circle or square represents a positive mHam, while an open circle or square represents a negative test. Patients in which only a single variant was identified are represented with circles, while patients with more than one variant are represented with squares. Each cohort is designated by a unique color (legend). The total number of amino acids (aa) in each protein are provided. Numbered protein domains denote complement control protein modules (CCPs), other protein domains are as labeled. HELLP, hemolysis, elevated liver enzymes, and low platelets; aHUS, atypical hemolytic uremic syndrome; SRCR, scavenger receptor cysteine-rich; LDL, low-density lipoprotein; MG, macroglobulin; ANA, anaphylatoxin; TM, transmembrane.

In our cohort, HELLP syndrome participants were associated with more frequent adverse maternal and fetal outcomes compared with the partial HELLP syndrome or HPC participants. Women with HELLP syndrome are more likely to have increased morbidity and mortality secondary to disseminated intravascular coagulation and hepatic rupture compared with other hypertensive disorders of pregnancy $(28,29)$. However, 6 of the 7 women with HELLP syndrome who had maternal adverse events were either positive by the mHam assay or had a rare variant in an APC gene. Our data suggest that the increased morbidity or mortality in HELLP syndrome is associated with dysregulation of the APC. In the partial HELLP group, there was only one participant (PH6) with an adverse maternal outcome (an eclamptic seizure); she was also positive in the 
Table 4. aHUS and TTP participants

\begin{tabular}{|c|c|c|c|c|c|c|c|c|c|c|}
\hline ID & Race & Age (yr) & $\mathrm{Cr}(\mathrm{mg} / \mathrm{dl})$ & $\begin{array}{l}\text { Highest AST } \\
\text { (IU/I) }\end{array}$ & $\begin{array}{c}\text { Highest ALT } \\
\text { (IU/I) }\end{array}$ & $\begin{array}{c}\text { Lowest } \\
\text { Platelets } \\
\left(\mathbf{m m}^{3}\right)\end{array}$ & $\begin{array}{l}\text { Highest LDH } \\
\text { (IU/L) }\end{array}$ & mHAM (\%) & $\begin{array}{l}\text { ADAMTS13 } \\
(\%)\end{array}$ & $\begin{array}{l}\text { Germline } \\
\text { Variant }\end{array}$ \\
\hline TTP1 & White & 38 & 0.87 & 61 & 37 & 7 & 1,081 & 23 & $<5$ & None \\
\hline TTP3 & Black & 32 & 2.3 & 473 & 187 & 4 & 2,215 & 17 & $<5$ & None \\
\hline TTP4 & Black & 43 & 1.3 & 58 & 41 & 4 & 1,985 & 16 & $<5$ & None \\
\hline TTP7 & Black & 60 & 1.9 & 30 & 27 & 9 & 2,337 & 5 & $<10$ & None \\
\hline TTP8 & Black & 64 & 1.2 & 53 & 13 & 10 & 1,482 & $\mathrm{n} / \mathrm{a}$ & $<5$ & None \\
\hline aHUS1 & Black & 47 & 4.3 & 28 & 22 & 17 & 1,718 & 63 & 88 & $\begin{array}{c}\text { CFB p.I242L, } \\
\text { del(CFHR1) } \\
\text { homozygous }\end{array}$ \\
\hline aHUS4 & Black & 38 & 6.1 & 47 & 20 & 63 & 917 & 39 & $>100$ & $\begin{array}{l}\text { CFH p.V158I, } \\
\text { CFH p.A1027P }\end{array}$ \\
\hline aHUS5 & White & 38 & 3.48 & 93 & 52 & 45 & 2,501 & 39 & $>100$ & THBD p.A43T \\
\hline aHUS6 & White & 32 & 6.5 & 26 & 15 & 76 & 495 & 28 & 96 & CD46 p.W216C \\
\hline aHUS7 & Asian & 32 & 7.2 & 109 & 106 & 7 & 1,588 & 28 & 41 & $\begin{array}{l}\text { CFHR5 } \\
\text { p.P453A }\end{array}$ \\
\hline aHUS8 & White & 25 & $\mathrm{n} / \mathrm{a}$ & $\mathrm{n} / \mathrm{a}$ & $\mathrm{n} / \mathrm{a}$ & $\mathrm{n} / \mathrm{a}$ & $\mathrm{n} / \mathrm{a}$ & 27 & $\mathrm{n} / \mathrm{a}$ & CFH p.R1078S \\
\hline aHUS9 & White & 78 & 4 & 86 & 32 & 47 & 1,473 & 26 & 53 & $\begin{array}{c}\text { del(CFHR1- } \\
\text { CFHR3) } \\
\text { homozygous }\end{array}$ \\
\hline aHUS10 & White & 51 & 3.7 & 63 & 20 & 18 & 5,266 & 25 & $>100$ & $\begin{array}{c}\text { CFH p.R1074P, } \\
\text { CD46 } \\
\text { p.S274YFS*11 }\end{array}$ \\
\hline aHUS16 & White & 25 & 1.4 & 46 & 18 & 20 & 1,160 & 10 & 82 & None \\
\hline aHUS17 & White & 61 & 1.6 & 75 & 85 & 21 & 549 & 9 & 87 & None \\
\hline aHUS18 & White & 17 & $\mathrm{n} / \mathrm{a}$ & $\mathrm{n} / \mathrm{a}$ & $\mathrm{n} / \mathrm{a}$ & $\mathrm{n} / \mathrm{a}$ & $\mathrm{n} / \mathrm{a}$ & $\mathrm{n} / \mathrm{a}$ & 34 & None \\
\hline
\end{tabular}

aHUS, atypical hemolytic uremic syndrome; TTP, thrombotic thrombocytopenic purpura; yr, years; Cr, creatinine; AST, aspartate aminotransferase; ALT, alanine aminotransferase; LDH, lactate dehydrogenase; mHam, modified Ham.

mHam, indicating increased APC activation. There was one participant in the HPC group with a negative mHam and genetic analysis who was later diagnosed with preeclampsia.

In the HELLP group there were 7 participants who had adverse fetal outcomes, 3 of which were terminations of pregnancy secondary to early gestational age and fetal weight less than 500 grams at time of diagnosis. Two of these had either a positive mHam or a rare germline variant. In the partial HELLP group there were 4 participants with adverse fetal outcomes, all of whom had either a positive mHam test or a rare germline variant, further indicating that abnormal complement regulation is associated with both poor maternal and fetal outcomes. There were 2 HPC participants with positive mHam assays, potentially secondary to the normal upregulation of the complement system during pregnancy, particularly in the third trimester (9). Further, there were 2 HPC participants with rare variants of the APC. Similar to multiple other genetic diseases (hemochromatosis, for example), rare variants of the APC are associated with incomplete penetrance and variable expressivity (30-35). Therefore, genetic 
Table 5. Comparison of mHam for all groups

\begin{tabular}{lcccccc}
\hline Participant Group & $(+)$ mHam (\%) & (-) mHam (\%) & Participant Group & (+) mHam (\%) & $(-)$ mHam (\%) & P value \\
HELLP Syndrome & $8(62 \%)$ & $5(38 \%)$ & HPC & $2(11 \%)$ & $16(89 \%)$ & 0.006 \\
HELLP Syndrome & $8(62 \%)$ & $5(38 \%)$ & HPC+TTP & $4(16 \%)$ & $21(84 \%)$ & 0.009 \\
aHUS & $15(88 \%)$ & $2(12 \%)$ & HPC+TTP & $4(16 \%)$ & $21(84 \%)$ & $<0.001$ \\
HELLP Syndrome & $8(62 \%)$ & $5(38 \%)$ & aHUS & $15(88 \%)$ & $2(12 \%)$ & 0.19
\end{tabular}

HELLP, hemolysis, elevated liver enzymes, and low platelets; mHam, modified Ham assay; aHUS, atypical hemolytic uremic syndrome; HPC, healthy pregnant control; TTP, thrombotic thrombocytopenic purpura.

variants are best characterized as risk factors, with disease onset occurring in the setting of additional environmental stress causing complement activation.

Our findings have important clinical implications for the diagnosis and treatment of HELLP syndrome. Reliable biomarkers and genetic mutations that predispose to disease states are critical for planning future clinical trials and for targeted approaches to therapy. Paroxysmal nocturnal hemoglobinuria $(\mathrm{PNH})$ and aHUS are diseases caused by mutations that predispose cells to complementmediated destruction $(16,36-38)$. Both of these conditions are dramatically and rapidly improved by blocking terminal complement with the humanized monoclonal antibody, eculizumab (22, 39-41). Prior to FDA approval of eculizumab, pregnancy was relatively contraindicated in PNH due to excessive complement activation that increased fetal and maternal mortality. Eculizumab is now routinely recommended for $\mathrm{PNH}$ patients who become pregnant since the drug does not effectively cross the placenta or enter the breast milk (42). Complement inhibition has also greatly improved the outcome of patients with aHUS. Recent studies in aHUS show that this treatment can be safely discontinued in most patients once a complete remission is achieved and the complement trigger has resolved (43). Our study confirms that aHUS and HELLP share similar pathophysiology. Indeed, there is a case report of eculizumab treatment leading to clinical improvement and prolongation of pregnancy in a patient with HELLP syndrome (44). Furthermore, we previously demonstrated that C5 inhibition can effectively block complement-mediated killing in HELLP serum in vitro (13). Currently, HELLP syndrome is most effectively managed by delivering the fetus (removal of the complement trigger); however, in cases of early gestational age (before 28 weeks) there is a high risk of fetal mortality and maternal morbidity (1). Based on our data and those of others, we suggest that future trials of complement inhibition in HELLP syndrome include genetic testing and the mHam assay to firmly establish the diagnosis. Patients with HELLP syndrome diagnosed prior to 28 weeks gestational age may benefit from this therapeutic intervention to allow the fetus to reach a safer gestational age and achieve less neonatal morbidity.

In summary, most HELLP syndrome and likely some preeclampsia, is driven by failure to regulate the APC. Serologic assays demonstrating a reduced ability to regulate complement (mHam), germline mutations in genes that regulate the APC, or both, are found in a majority of patients with HELLP syndrome. While we do not advocate for off-label use of complement inhibitors in HELLP syndrome, our findings form the basis for the design of clinical trials to test the efficacy of complement inhibition to reduce perinatal morbidity from early prematurity.

Table 6. Comparison of germline mutation status for all groups

\begin{tabular}{lcccccc}
\hline Participant Group & (+) mutation $^{\mathrm{A}}(\%)$ & (-) mutation (\%) & Participant Group & (+) mutation (\%) & (-) mutation (\%) & P value \\
HELLP Syndrome & $5(46 \%)$ & $6(54 \%)$ & HPC & $2(12 \%)$ & $15(88 \%)$ & 0.07 \\
HELLP Syndrome & $5(46 \%)$ & $6(54 \%)$ & HPC+TTP & $2(8 \%)$ & $23(92 \%)$ \\
aHUS & $10(56 \%)$ & $8(44 \%)$ & HPC+TTP & $2(8 \%)$ & $23(92 \%)$ \\
HELLP Syndrome & $5(46 \%)$ & $6(54 \%)$ & aHUS & $10(56 \%)$ & $8(44 \%)$ & 0.01 \\
\end{tabular}

${ }^{A}$ Mutation = rare germline variant in a gene of the alternative pathway of complement. HELLP, hemolysis, elevated liver enzymes, and low platelets; aHUS, atypical hemolytic uremic syndrome; HPC, healthy pregnant control; TTP, thrombotic thrombocytopenic purpura. 
Table 7. Germline variant characteristics

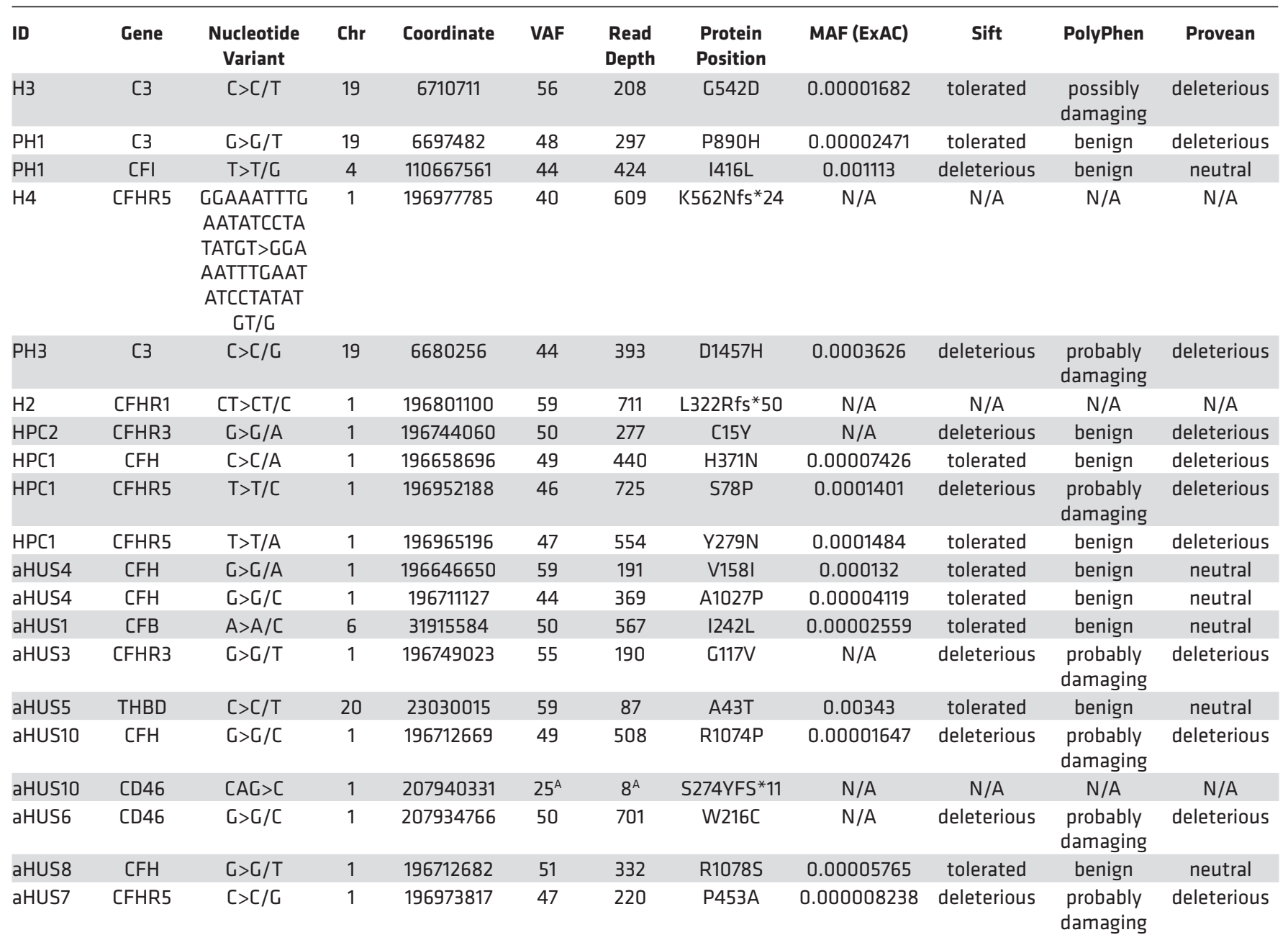

AThis variant did not meet our filtering criteria; however, it has been previously reported (39) and verified via CLIA laboratory testing. Chr, chromosome; VAF, variant allele frequency; MAF, minor allele frequency as reported in the ExAC database.

\section{Methods}

Study population and design. We performed a prospective case-controlled study to identify whether HELLP syndrome is associated with both activation of the APC as well as germline variants in genes that regulate the APC. We hypothesized that HELLP syndrome patients harboring genetic variants of APC genes would also display activation of the APC via a functional assay (the mHam). All participants were recruited from the Johns Hopkins Medical Institute, and none had been studied previously. The Tennessee criteria were used for the diagnosis of HELLP syndrome, and partial HELLP syndrome was defined by having 1 or 2 but not all lab abnormalities of HELLP syndrome (Supplemental Table 1; supplemental material available online with this article; https://doi.org/10.1172/jci.insight.99128DS1) $(1,45)$. We recruited all participants from November 1, 2015 to December 31, 2016. Healthy pregnant controls were recruited after 23 weeks of gestation, and were excluded if they had a history of hypertensive disease, complement or microangiopathic disorder, or autoimmune disease. Blood was collected from all participants at the time of recruitment by venipuncture of the antecubital vein. Serum was cold centrifuged within 2 hours of collection at $4^{\circ} \mathrm{C}$ and stored at $-80^{\circ} \mathrm{C}$. Whole blood was used to generate genomic DNA for targeted gene sequencing using a Qiagen DNeasy Blood \& Tissue Kit. All samples were deidentified and coded prior to laboratory testing. Patients with aHUS were recruited using standard diagnostic criteria defined by Legendre et al. (39). Patients with TTP were recruited following confirmation of the diagnosis via an ADAMTS13 activity less than $10 \%$. 
mHam assay. The mHam assay was performed as previously described (20). Briefly, PNH-like (PIGA-null) TF-1 cells were used to assess complement-mediated killing via the APC. Cells were plated in U-shaped wells at a density at 4,000 cells/well and incubated in 20\% serum with Gelatin Veronal Buffer (GVB, Complement Technology, Inc). Each sample was tested in triplicate for 30 minutes at $37^{\circ} \mathrm{C}$. After incubation, the supernatant was removed and cell proliferation agent WST-1 (Roche) was added and incubated for 2 hours at $37^{\circ} \mathrm{C}$. Absorbance was measured in an iMark Microplate Absorbance Reader (Bio-Rad) at $490 \mathrm{~nm}$ with a reference wave length at $595 \mathrm{~nm}$. Heat-inactivated serum was used as a negative control for each sample. Heat inactivation was performed by incubating the serum at $56^{\circ} \mathrm{C}$ for 30 minutes. Normal human AB serum (H4522, Sigma-Aldrich) was used as an internal control in the assay. The percentage of live cells was calculated as the ratio of the absorbance of each sample divided by the absorbance of the same sample's heat-inactivated control. The percentage of nonviable cells (cell killing) was calculated as 1 minus this ratio.

Targeted sequencing. A custom panel of 9 genes known to function in the APC was selected for sequencing using the Illumina TruSeq Custom Amplicon (v1.5). Illumina DesignStudio software was utilized to generate custom oligonucleotides designed to amplify exonic regions of the following selected genes: CFH, CFB, CFI, CFHR1, CFHR3, CFHR5, C3, CD46 (MCP), and THBD. The panel consisted of 402 amplicons covering a total of $58.3 \mathrm{~kb}$ with an overall coverage of $98 \%$. An amplicon library of each patient sample was generated according to the manufacturer's protocol using $250 \mathrm{ng}$ genomic DNA as input. Successful library amplification was verified for a subset of samples by both gel electrophoresis and Bioanalyzer (Agilent) analysis. This was followed by library normalization and pooling per manufacturer protocol, and paired-end sequencing on the Illumina MiSeq platform via the Genetic Resources Core Facility (GRCF) at Johns Hopkins School of Medicine (https://grcf.jhmi.edu). Analysis of raw sequencing data (FASTQ) was performed using the TruSeq Amplicon application (v2.0.0) via the Illumina BaseSpace platform, aligning to (GRCh37/hg19) human genome reference. Average base coverage was $377 \times$ per sample. SNVs and small indels were identified using Isaac variant caller and variant call files were filtered using VariantStudio software (v3.0). Variants not passing Illumina's variant quality filters were excluded. The following criteria were used to identify rare germline SNVs and indels: nonsynonymous coding region variants with a depth greater than $20 \times$, variant allele frequency between $40 \%$ and $60 \%$, Exome Aggregation Consortium (ExAC v0.3.1) minor allele frequency less than 0.01 in any ethnic population (total of 60,706 samples), and minor allele frequency less than 0.01 in the Exome Variant Server (46). Large deletions were determined by complete loss of signal for multiple consecutive amplicons (Supplemental Figure 1). All genomic coordinates refer to hg19.

Adverse events. The investigators sought to observe any adverse maternal or fetal events in the HELLP group, partial HELLP group, and HPC group. Maternal adverse events were maternal mortality, intensive care unit admission, renal failure, blood product transfusion, and eclampsia in HELLP or partial HELLP groups, or new diagnosis of preeclampsia in a control group. Fetal adverse events were fetal termination or abortion secondary to diagnosis of HELLP or partial HELLP syndrome, neonatal mortality, and fetal growth restriction. Other than worsening of HELLP syndrome in the setting of a nonviable fetus, no other factors were relevant for fetal terminations. Because neonatal intensive care unit admission in preeclampsia and HELLP syndrome is usually secondary to iatrogenic preterm birth, we did not include this as an adverse event in our group.

Statistics. A 1-way ANOVA test with Bonferroni's correction was used to calculate the $P$ values for the difference among the means for age, BMI, estimated gestational age in HELLP, partial HELLP, and the HPC groups. Either $\chi^{2}$ test or Fisher's exact test was used to calculate the rest of the $P$ values provided. A Fisher's exact was used if more than $20 \%$ of the expected values in the input cells were less than 5 and/or any individual values had expected counts of less than 1 . Statistical significance for the mHam assay was calculated as a binary value (positive or negative), as the mHam assay is thought to be clinically significant as a qualitative test. All analyses were performed by using SPSS 18 for Windows/MAC (PASW Statistics GradPack 18; SPSS). All tests were 2-sided with $P$ less than 0.05 indicating significance.

Study approval. This study was approved by the Johns Hopkins institutional review board and conducted according to Declaration of Helsinki principles. Written informed consent was received from all participants prior to inclusion in the study. 


\section{Author contributions}

AJV and RAB were responsible for the study conception and design. AJV, EMB, RAB, SAM, SC, SE, KB, and CJS collected and categorized study participants. AJV, EMB, XY, IM, and ACB performed experiments and acquired data. AJV, EMB, and RAB analyzed and interpreted the data. JJ performed statistical data analysis. AJV, EMB, and RAB drafted the manuscript and performed critical revisions. All authors approved the final version of the manuscript.

\section{Acknowledgments}

AJV is awarded the Johns Hopkins Meyerhoff Professorship Award and T32HL007525 NIH/NHLBI Training Program in Hematology. RAB is awarded the R01HL133113 NIH/NHLBI Complementopathies: Genotype and Phenotype. ACB, who is currently employed at the FDA, was involved with this study during a previous affiliation with Johns Hopkins School of Medicine. The views expressed in this paper do not represent the views of the FDA.

Address correspondence to: Robert A. Brodsky, Ross Research Building, Room 1025, Johns Hopkins University School of Medicine, 720 Rutland Avenue, Baltimore, Maryland 21205, USA. Phone: 410.502.2546; Email: rbrodsky@jhmi.edu.

ACB's present address is: FDA, Silver Spring, Maryland, USA.

1. Report of the American College of Obstetricians Gynecologists' Task Force on Hypertension in Pregnancy. Hypertension in pregnancy: Executive Summary. Obstet Gynecol. 2013;122(5):1122-1131.

2. Chen XK, Wen SW, Smith GN, Yang Q, Walker MC. New-onset hypertension in late pregnancy and fetal growth: different associations between singletons and twins. Hypertens Pregnancy. 2007;26(3):259-272.

3. Iacobelli S, Bonsante F, Robillard PY. Pre-eclampsia and preterm birth in Reunion Island: a 13 years cohort-based study. Comparison with international data. J Matern Fetal Neonatal Med. 2016;29(18):3035-3040.

4. Weinstein L. Syndrome of hemolysis, elevated liver enzymes, and low platelet count: a severe consequence of hypertension in pregnancy. 1982. Am J Obstet Gynecol. 2005;193(3 Pt 1):859.

5. Fitzpatrick KE, Hinshaw K, Kurinczuk JJ, Knight M. Risk factors, management, and outcomes of hemolysis, elevated liver enzymes, and low platelets syndrome and elevated liver enzymes, low platelets syndrome. Obstet Gynecol. 2014;123(3):618-627.

6. Uzan J, Carbonnel M, Piconne O, Asmar R, Ayoubi JM. Pre-eclampsia: pathophysiology, diagnosis, and management. Vasc Health Risk Manag. 2011;7:467-474.

7. Martin JN, Rose CH, Briery CM. Understanding and managing HELLP syndrome: the integral role of aggressive glucocorticoids for mother and child. Am J Obstet Gynecol. 2006;195(4):914-934.

8. Martin JN, Rinehart BK, May WL, Magann EF, Terrone DA, Blake PG. The spectrum of severe preeclampsia: comparative analysis by HELLP (hemolysis, elevated liver enzyme levels, and low platelet count) syndrome classification. Am J Obstet Gynecol. 1999;180(6 Pt 1):1373-1384.

9. Richani K, et al. Normal pregnancy is characterized by systemic activation of the complement system. J Matern Fetal Neonatal Med. 2005;17(4):239-245.

10. Haeger M, Unander M, Bengtsson A. Enhanced anaphylatoxin and terminal C5b-9 complement complex formation in patients with the syndrome of hemolysis, elevated liver enzymes, and low platelet count. Obstet Gynecol. 1990;76(4):698-702.

11. Fakhouri F, et al. Pregnancy-associated hemolytic uremic syndrome revisited in the era of complement gene mutations. $J A m$ Soc Nephrol. 2010;21(5):859-867.

12. Loirat C, Frémeaux-Bacchi V. Atypical hemolytic uremic syndrome. Orphanet J Rare Dis. 2011;6:60.

13. Vaught AJ, et al. Direct evidence of complement activation in HELLP syndrome: A link to atypical hemolytic uremic syndrome. Exp Hematol. 2016;44(5):390-398.

14. Salmon JE, et al. Mutations in complement regulatory proteins predispose to preeclampsia: a genetic analysis of the PROMISSE cohort. PLoS Med. 2011;8(3):e1001013.

15. Noris M, et al. Relative role of genetic complement abnormalities in sporadic and familial aHUS and their impact on clinical phenotype. Clin J Am Soc Nephrol. 2010;5(10):1844-1859.

16. Noris M, Bresin E, Mele C, Remuzzi G. Genetic atypical hemolytic-uremic syndrome. In: Pagon RA, et al., eds. GeneReviews(R). Seattle, WA: University of Washington, Seattle; 1993. URL: https://www.ncbi.nlm.nih.gov/books/NBK1367. Accessed February 27, 2018.

17. Maga TK, Nishimura CJ, Weaver AE, Frees KL, Smith RJ. Mutations in alternative pathway complement proteins in American patients with atypical hemolytic uremic syndrome. Hum Mutat. 2010;31(6):E1445-E1460.

18. Crovetto F, et al. The genetics of the alternative pathway of complement in the pathogenesis of HELLP syndrome. JMatern Fetal Neonatal Med. 2012;25(11):2322-2325.

19. Fakhouri F, et al. Factor H, membrane cofactor protein, and factor I mutations in patients with hemolysis, elevated liver enzymes, and low platelet count syndrome. Blood. 2008;112(12):4542-4545.

20. Gavriilaki E, et al. Modified Ham test for atypical hemolytic uremic syndrome. Blood. 2015;125(23):3637-3646.

21. Zipfel PF, et al. Deletion of complement factor H-related genes CFHR1 and CFHR3 is associated with atypical hemolytic uremic 
syndrome. PLoS Genet. 2007;3(3):e41.

22. Legendre CM, Licht C, Loirat C. Eculizumab in atypical hemolytic-uremic syndrome. N Engl J Med. 2013;369(14):1379-1380.

23. Lynch AM, Wagner BD, Giclas PC, West NA, Gibbs RS, Holers VM. The relationship of longitudinal levels of complement Bb during pregnancy with preeclampsia. Am J Reprod Immunol. 2016;75(2):104-111.

24. Romem Y, Artal R. C-reactive protein in pregnancy and in the postpartum period. Am J Obstet Gynecol. 1985;151(3):380-383.

25. Ernst GD, et al. C-reactive protein levels in early pregnancy, fetal growth patterns, and the risk for neonatal complications: the Generation R Study. Am J Obstet Gynecol. 2011;205(2):132.e1-132.12.

26. Watts DH, Krohn MA, Wener MH, Eschenbach DA. C-reactive protein in normal pregnancy. Obstet Gynecol. 1991;77(2):176-180.

27. Derzsy Z, Prohászka Z, Rigó J, Füst G, Molvarec A. Activation of the complement system in normal pregnancy and preeclampsia. Mol Immunol. 2010;47(7-8):1500-1506.

28. Sibai BM. Diagnosis, controversies, and management of the syndrome of hemolysis, elevated liver enzymes, and low platelet count. Obstet Gynecol. 2004;103(5 Pt 1):981-991.

29. Sibai BM, Ramadan MK, Usta I, Salama M, Mercer BM, Friedman SA. Maternal morbidity and mortality in 442 pregnancies with hemolysis, elevated liver enzymes, and low platelets (HELLP syndrome). Am J Obstet Gynecol. 1993;169(4):1000-1006.

30. Richards A, et al. Implications of the initial mutations in membrane cofactor protein (MCP; CD46) leading to atypical hemolytic uremic syndrome. Mol Immunol. 2007;44(1-3):111-122.

31. Fremeaux-Bacchi V, et al. Genetic and functional analyses of membrane cofactor protein (CD46) mutations in atypical hemolytic uremic syndrome. J Am Soc Nephrol. 2006;17(7):2017-2025.

32. Esparza-Gordillo J, et al. Insights into hemolytic uremic syndrome: segregation of three independent predisposition factors in a large, multiple affected pedigree. Mol Immunol. 2006;43(11):1769-1775.

33. Esparza-Gordillo J, et al. Predisposition to atypical hemolytic uremic syndrome involves the concurrence of different susceptibility alleles in the regulators of complement activation gene cluster in 1q32. Hum Mol Genet. 2005;14(5):703-712

34. Papanikolaou G, et al. Genetic heterogeneity underlies juvenile hemochromatosis phenotype: analysis of three families of northern Greek origin. Blood Cells Mol Dis. 2002;29(2):168-173.

35. Piperno A, et al. Heterogeneity of hemochromatosis in Italy. Gastroenterology. 1998;114(5):996-1002.

36. Hill A, DeZern AE, Kinoshita T, Brodsky RA. Paroxysmal nocturnal haemoglobinuria. Nat Rev Dis Primers. 2017;3:17028.

37. Brodsky RA. Complement in hemolytic anemia. Blood. 2015;126(22):2459-2465.

38. Brodsky RA. Paroxysmal nocturnal hemoglobinuria. Blood. 2014;124(18):2804-2811

39. Legendre CM, et al. Terminal complement inhibitor eculizumab in atypical hemolytic-uremic syndrome. $N E n g l J M e d$. 2013;368(23):2169-2181.

40. Brodsky RA, et al. Multicenter phase 3 study of the complement inhibitor eculizumab for the treatment of patients with paroxysmal nocturnal hemoglobinuria. Blood. 2008;111(4):1840-1847.

41. Hillmen P, et al. The complement inhibitor eculizumab in paroxysmal nocturnal hemoglobinuria. $N$ Engl J Med. 2006;355(12):1233-1243.

42. Kelly RJ, et al. Eculizumab in Pregnant Patients with Paroxysmal Nocturnal Hemoglobinuria. N Engl J Med. 2015;373(11):1032-1039.

43. Merrill SA, Brittingham ZD, Yuan X, Moliterno AR, Sperati CJ, Brodsky RA. Eculizumab cessation in atypical hemolytic uremic syndrome. Blood. 2017;130(3):368-372

44. Burwick RM, Feinberg BB. Eculizumab for the treatment of preeclampsia/HELLP syndrome. Placenta. 2013;34(2):201-203.

45. Aydin S, Ersan F, Ark C, Arığlu Aydın C. Partial HELLP syndrome: maternal, perinatal, subsequent pregnancy and long-term maternal outcomes. J Obstet Gynaecol Res. 2014;40(4):932-940.

46. Lek M, et al. Analysis of protein-coding genetic variation in 60,706 humans. Nature. 2016;536(7616):285-291. 